\title{
Market Structure and Schumpeterian Growth
}

\author{
Val E. Lambson \\ Department of Economics \\ P.O. Box 22363 \\ Brigham Young University \\ Provo, UT 84602-2363 \\ phone: (801) 422-7765 \\ fax: (801) 422-0194 \\ email: vel@byu.edu
}

\author{
Kerk L. Phillips \\ Department of Economics \\ P.O. Box 22363 \\ Brigham Young University \\ Provo, UT 84602-2363 \\ phone: (801) 422-5928 \\ fax: (801) 422-0194 \\ email: kerk_phillips@byu.edu
}

this version: $4 / 8 / 2004$

preliminary $\&$ incomplete, do not cite

Keywords: growth, market structure

JEL codes: L11, O31, O40 


\begin{abstract}
We present a discrete-time version of an otherwise standard Schumpeterian growth model. Unlike continuous-time models, with discrete time models, linear production functions for probabilities make no sense as they imply probabilities greater than one for sufficiently high inputs.

We show that in discrete time is possible for more than one firm to innovate simultaneously. How the profits are divided in the case of these ties is critical for aggregate behavior. Both a monopolist and a group of potential Bertrand competitors value an additional unit of $R \& D$ input only to the extent that it will succeed where all other inputs in the industry fail. Potentially collusive firms place the same value, but also value the success of their own inputs, even if another firm also succeeds.

This has implications for the choice a modeling framework. If ties are prevalent, models with linear functions for R\&D in continuous time, while tractable, will miss important behavior. We present evidence consistent with ties being prevalent in many industries.

In general equilibrium, where all prices are endogenously determined, the amount of $R \& D$ undertaken by a group of firms that engage in Bertrand competition when there is a tie is less than the amount undertaken if there is a single R\&D firm.

In general equilibrium, the amount of $R \& D$ undertaken by a group of firms that colludes when there is a tie is greater than the amount undertaken if there is a single $R \& D$ firm.
\end{abstract}




\section{Introduction}

Schumpeterian growth arises from the research and development (R\&D) activities of innovators pursuing the monopoly rents that accrue to new proprietary technologies. There is a large and insightful literature on Schumpeterian growth, including papers by Aghion and Howitt (1992), Grossman and Helpman (1991), and Segerstrom, Anant and Dinopoulos (1990). However, the Schumpeterian growth literature has thus far ignored the effects of post-innovation market structure when several innovators can be successful at once, that is, when ties are possible. It is easy to understand why: in the continuous time models that are typical in this literature, the probability of a tie is infinitesimal. Arguably, this ignores an important aspect of reality. $R \& D$ projects take time, and that time is naturally identified with the length of a discrete time period. If the period length is substantial then simultaneous (that is, same period) discoveries of similar innovations are likely to be common. ${ }^{1}$

Whether the additional complexity of modeling ties is worthwhile depends on how important they are empirically. If they rarely occur in the real world, then there is little to be lost in using continuous time models and the fact that their solutions are more tractable makes them very attractive. However, if ties occur frequently then the tractability may not be worth the cost.

We use data on markups and on the average growth rates of Solow residuals from Phillips (1993) to examine the importance of ties. The data are at the two-digit SIC level. Assume these industries grow in discrete jumps of size $\theta$, equal to the gross markups, with constant probabilities, $\rho$. We can solve for the probabilities of success in R\&D by using $g=\rho(\theta-1)$.

Table 1 presents the values of $\mu$ and $\theta$ for the sixteen industries where markups are greater than one and the implied values for $\rho$. Given $\rho$, we can calculate the probability of a tie given an innovation occurs, assuming $J$ equally sized firms. This is

\footnotetext{
${ }^{1}$ A famous example of such a tie might be the simultaneous introduction of VHS and Beta video cassette recording technologies. Closer to home, readers of academic literature can probably think of numerous examples of similar ideas being published at about the same time.
} 
reported in the last column of Table 1 for various values of $J$. The results show that ties are likely to empirically important for most industries.

The remainder of the paper explores a discrete time, infinite-horizon model that is analogous to the continuous time models that dominate the Schumpeterian growth literature. Sections 2-4 respectively describe the three sectors of the model economy: innovators, producers, and consumers. Producers employ labor in the production of a consumption good with the current technology. In each period $J$ innovators come into existence and employ labor with the goal of discovering a labor-saving technology and supplanting the current producer or producers.

Section 5 presents partial equilibrium analysis, assuming the industry is small enough to take wages and interest rates as given. Curiously, aggregate $R \& D$ is the same when $J>1$ and ties result in (profit-dissipating) Bertrand behavior as when $J=1$. The distribution of $R \& D$ across $J>1$ innovators is indeterminate. Of course, such indeterminacies are common in constant-returns-to-scale models, but ours is not such a model. In discrete time, the probability of success cannot be globally linear in inputs. So the source of the indeterminacy must lie elsewhere. We introduce a notion, called constant returns to duplication, that is interpretable as having innovators decide how many independent experiments they are going to run simultaneously during the period. In the Bertrand case, an additional experiment is of value to an innovator only if it succeeds when all other experiments fail. This is true whether the innovator or its competitors are conducting the other experiments; indeed it is true whether or not the innovator has competitors. Since the marginal value of an experiment depends only on the number of experiments and not on which innovators are running them, the total equilibrium number of experiments is independent of the number of innovators. Thus, in parital equilibrium, the Bertrand case yields the same level of R\&D as the monopoly case. By contrast, if ties result in collusive behavior or, equivalently, if a monopoly is randomly granted to one of the successful (risk-neutral) firms, then the results differ from the Bertrand case. In the collusive case an experiment has value whenever it is successful, so the aggregate number of experiments is higher than in the Bertrand case. In summary, if the number of innovators exceeds one, allowing collusion induces higher growth. 
Section 6 extends the analysis of Section 5 with a simple general equilibrium model that makes wages and interest rates endogenous. If $J>1$, the real wage depends on whether there was a tie in the previous period because of the effect on market structure in the output market. As a result, the equivalence of Bertrand and monopolistic behavior does not carry over from the partial equilibrium scenario. Section 6 also considers the welfare properties of the various market structures. As in the previous literature - see, for example, Aghion and Howitt (1998) - welfare effects are ambiguous. In all cases growth may be either too rapid or too slow, so it may or may not be optimal to allow collusion to increase the growth rate. Simulations suggest that, for reasonable parameters, the Bertrand outcome exhibits insufficient growth, suggesting that allowing collusion in the event of ties may be welfare-enhancing. Indeed, growth is substantially increased toward - but not beyond - the optimum even if there are only two innovators. There is an optimal number of innovators such that allowing collusion yields growth close to the socially optimal level. This optimal number of innovators is rather small. Finally, the welfare loss is asymmetric in the sense that the loss associated with allowing collusion and overshooting the optimal number of innovators by as much as nearly a thousand is significantly smaller than the loss associated with imposing Bertrand competition.

Section 7 contains some concluding remarks.

\section{Innovators}

Consider an industry that uses labor to produce a consumption good. There are countably infinitely many time periods indexed by the positive integers. The prevailing technology in period $t$ is characterized by its (constant) output per worker, $A_{t}>0$. At the beginning of each period $J \geq 1$ innovators are born. The innovators employ labor in hopes of discovering a new technology characterized by output per worker of $\theta A_{t}$ where $\theta>1$. If an innovator hires $\ell$ workers, it has a probability $\rho(\ell)$ of discovering the new technology. Successful innovators (if any) cease research and begin production in period $t+1$. Unsuccessful innovators cease to exist.

A constant returns to scale assumption is widely imposed in the Schumpeterian growth literature. Specifically, it is assumed that $\rho$ is homogeneous of degree one over 
input levels satisfying $\rho \in(0,1)$. Of course, constant returns cannot hold globally because $\rho$ is bounded above by one. On the other hand, the intuition behind constant returns - that proportional increases in inputs should lead to (at least) a proportional increase in output - is compelling. We reconcile this logic with its failure to hold globally when applied to $\rho$ by taking the view that it is misleading to think of $\rho$ as a typical production function. In our view, $R \& D$ underwrites experiments which may be successful or unsuccessful. It is then natural to assume that an increase in inputs results in a proportional increase in the number of experiments underwritten. The properties of $\rho$ can then be deduced from the properties of the stochastic outcomes of the experiments. The length of a time period is naturally identified with the time required to run an experiment, and the innovator's choice of labor dictates how many experiments it can run simultaneously.

A natural analog to constant returns to scale, which we call constant returns to duplication, posits that all experiments have the same required labor input and the same (independent) probabilities of success. Let $\phi$ be the amount of labor required to conduct one experiment. Then the number of successes in $x$ experiments (requiring labor input of $\ell=x \phi$ ) is binomially distributed. As is well known, this distribution is approximately a Poisson distribution if $\phi$ is small. Specifically, if an innovator hires $\ell$ units of labor, the probability that it has at least one success - and hence discovers the new technology - is $\rho(\ell)=1-e^{-\kappa \ell}$, where $\kappa>0$.

Now consider the problem facing each of the $J$ innovators in each period $t$. Let $\ell_{j t}$ be the labor employed by innovator $j$ in period $t$, let $\ell_{t}=\left(\ell_{1 t}, \ldots, \ell_{J t}\right)$, let $\ell_{-j t}$ be $\ell_{t}$ with the $j^{\text {th }}$ element removed, and let $\tilde{\ell}_{t+1}=\left(\ell_{t+1}, \ell_{t+2}, \ldots\right){ }^{2}$ Given (possibly stochastic) sequences of wage rates $\widetilde{w}_{1}=\left(w_{1}, w_{2}, \ldots\right)$ and interest rates $\widetilde{r}_{1}=\left(r_{1}, r_{2}, \ldots\right)$, a Nash equilibrium for innovators is a sequence $\tilde{\ell}_{1}^{e}=\left(\ell_{1}^{e}, \ell_{2}^{e}, \ldots\right)$ such that, for all innovators $j$ in all time periods $t$,

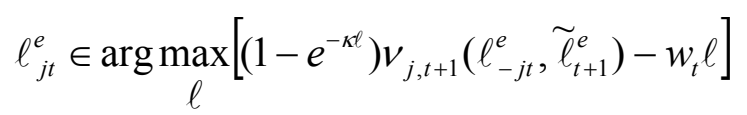

\footnotetext{
${ }^{2}$ For any variable, say $x, \tilde{x}_{t}$ will denote a (possibly stochastic) sequence of values of $x$ beginning in period $t$.
} 
where $v_{j, t+1}\left(\ell_{-j t}^{e}, \tilde{\ell}_{t+1}^{e}\right)$ is the expected present value of the profit stream - discounted to period $t$ - associated with introducing an innovation in period $t+1$. The first order condition associated with (2.1) - assuming an interior solution - is

$$
\kappa e^{-\kappa \ell} v_{j, t+1}\left(\ell_{-j t}^{e}, \tilde{\ell}_{t+1}^{e}\right)-w_{t}=0
$$

Note that $v_{j, t+1}$ depends on $\ell_{-j t}^{e}$ because this determines how many competitors the successful innovator can expect to face, that is, how many other successful innovators from period $t$ there will be. Note also that $v_{j, t+1}$ depends on $\tilde{\ell}_{t+1}^{e}$ because this determines how long the successful innovator in period $t$ will enjoy the lead in technology. The properties of $v_{j, t+1}$ will be derived below.

\section{Producers}

In each period, $t$, producers - that is, the most recently successful innovators enjoy a state-of-the-art technology with constant marginal cost $w_{t} / A_{t}$. Producers simultaneously choose prices to maximize current profits. They face competition from each other as well as from one or more of the previous producers that have the next oldest technology with marginal cost $w_{t} \theta / A_{t}$, where $\theta>1$. If in period $t$ there is a single producer, or if there are several producers who can collude, they charge the previous producers' marginal cost. ${ }^{3}$ This keeps the previous producers out of the market and earns aggregate profit of $\pi_{t}=(\theta-1) w_{t} C_{t} / A_{t}=(\theta-1) P_{t} C_{t} / \theta$, where $C_{t}$ is the quantity demanded. By contrast, if there are several producers who are unable to collude, they charge their own marginal cost, $P_{t}=w_{t} / A_{t}$, and earn aggregate profit $\pi_{t}=0$ in the resulting Bertrand equilibrium. Producers in the aggregate employ $N_{t} \equiv C_{t} / A_{t}$ units of labor.

The properties of $v_{j, t+1}$, the expected present value of the profit stream associated with introducing an innovation in period $t+1$, can now be described. A successful innovator in period $t$ has the most efficient technology in period $t+1$ with probability

\footnotetext{
${ }^{3}$ This assumes that profits are increasing in price below $w_{t} \theta / A_{t}$. Such is the case given the assumptions about consumers imposed below.
} 
$\gamma_{t+1}=1$. Thereafter, the probability that its technology remains the most efficient in period $\tau$ conditional on having been the most efficient in period $\tau-1$ equals the probability that all innovators fail in period $\tau-1$ :

$$
\gamma_{\tau}=\prod_{j=1}^{J} e^{-\kappa \ell_{j, \tau-1}}=e^{-\kappa \sum_{j=1}^{J} \ell_{j, \tau-1}}
$$

Thus, conditional on being successful, an innovator discounts profits in period $s$ back to period $t$ by the factor

$$
\delta_{s} \equiv \frac{1}{1+r_{t}} \prod_{\tau=t+1}^{s} \frac{\gamma_{\tau}}{1+r_{\tau}}
$$

which incorporates the probability of survival as well as the interest rate.

When $J=1$, if the sole innovator in period $t$ is successful it becomes a monopolist until it is supplanted by a new successful innovator. The associated value of $v_{t+1}$ - to be denoted $v_{t+1}^{s}-$ is

$$
v_{t+1}^{s}=\sum_{s=t+1}^{\infty} \delta_{s}(\theta-1) w_{s} C_{s} / A_{t+1}
$$

When $J>1, R \& D$ ties sometimes occur. Suppose, given a tie, successful innovators become Bertrand competitors thereafter. A successful innovator in period $t$ enjoys no unless all other innovators fail. This probability is $\prod_{i \neq j} e^{-\kappa \ell_{i t}}=e^{-\kappa \Sigma_{i \neq} \ell_{i i}}$. Thus in this case, the value $v_{j, t+1}$ - to be denoted $v_{j, t+1}^{b}-$ is ${ }^{4}$

$$
v_{j, t+1}^{b}=e^{-\kappa \Sigma_{i \neq j} \ell_{i t}} \sum_{s=t+1}^{\infty} \delta_{s}(\theta-1) w_{s} C_{s} / A_{t+1}
$$

Finally, suppose successful innovators collude ex post and that each has an equal share of the monopoly profit until a new technology is discovered. Then the value $v_{j, t+1}$ - to be denoted $v_{j, t+1}^{c}-$ is

$$
v_{j, t+1}^{c}=\sum_{U \in 2^{J-1}}\left[\alpha(U) \sum_{s=t+1}^{\infty} \frac{\delta_{s}(\theta-1) w_{s} C_{s}}{A_{t+1}(1+\# U)}\right]
$$

\footnotetext{
${ }^{4}$ Although $v_{t+1}^{b}$ can differ across firms, since firms may invest different amounts, the firm subscript is suppressed to simplify notation, and similarly for $\alpha(j)$ below.
} 
where $\alpha(U)$ is the probability that the set of successful innovators is $U, 2^{J-1}$ denotes the collection of subsets of innovators that do not include $j$, and $\# U$ is the number of successful innovators in set $U$. Note that $v_{j, t+1}^{c}$ can also be interpreted as the expected discounted payoff from successful innovation if a monopoly position - e.g. a patent - is granted randomly to one of the successful innovators.

\section{Consumers}

In each period, $t$, consumers divide their current income between consumption $C_{t}$, and savings in the form of a full set of Arrow-Debreu assets. Let $Q_{i t}$ denote the holdings of asset $i$ in period $t$, purchased in period $t-1$. With $J$ independent R\&D firms each either succeeding or failing there are $J^{2}$ different states of nature. Consumers' current income is comprised of the wage for their one unit of inelastically supplied labor, $w_{t}$, and the current value of the previous period's investments, $\Sigma_{i=1}^{J^{2}} d_{i t} Q_{i t}$.

Taking the stochastic sequence of wage rates, interest rates, output prices, and profits as given, consumers choose consumption and investments each period to maximize the expected present value of lifetime utility, $\sum_{t=1}^{\infty} \beta^{t-1} U\left(C_{t}\right)$, subject to the constraint that current expenditures on consumption and savings must equal current income for all $t$ and for all realizations of the stochastic process:

$$
P_{t} C_{t}=w_{t}+\sum_{i=1}^{J^{2}} d_{i t} Q_{i t}-\sum_{i=1}^{J^{2}} q_{i t} Q_{i t+1}
$$

where the $d_{i t}$ denotes the payoff of one unit of asset $i$ in period $t$ and the $q_{i t}$ denotes its price.

Subject to well-known regularity conditions, the consumers' problem can be solved recursively using the Bellman equation:

$$
V\left(\mathbf{Q}_{t}, \mathbf{\Omega}_{t}\right)=\max _{\mathbf{Q}_{t+1}} U\left(C_{t}\right)+E\left\{\beta\left[V\left(\mathbf{Q}_{t+1}, \mathbf{\Omega}_{t+1}\right)\right]\right\}
$$

where $C_{t}$ satisfies the budget constraint, $\mathbf{Q}_{t} \equiv\left\{Q_{i t}\right\}_{i=1}^{J^{2}}$ and $\Omega_{t}$ is the information set used to form expectations in period $t$. Standard dynamic programming techniques yield the consumers' necessary conditions for all $t$ and all realizations: 


$$
U^{\prime}\left(C_{t}\right) \frac{q_{i t}}{P_{t}}=\beta E\left\{\frac{d_{i t+1}}{P_{t+1}} U^{\prime}\left(C_{t+1}\right)\right\} ; \forall i
$$

In interest of tractability and comparability with the rest of the literature, we follow the common practice of restricting attention to the utility function $U\left(C_{t}\right)=\left(C_{t}^{1-\sigma}-1\right) /(1-\sigma)$ where $\sigma \in(0,1)$.

The market discount factor, $\frac{1}{1+r}$, is the intertemporal price of one unit of consumption tomorrow in terms of one unit of consumption today. Purchasing one of each available Arrow-Debreu assets gives exactly one unit of consumption with certainty. We use this fact, sum and manipulate the $I$ equations in (4.1) and get

$$
\frac{1}{1+r}=\beta E\left\{\frac{P_{t}}{P_{t+1}}\left(\frac{C_{t+1}}{C_{t}}\right)^{-\sigma}\right\}
$$

\section{Partial Equilibrium}

Consider a partial equilibrium model of a single $R \& D$ race where current wages are exogenous to the industry. A successful innovation by only one firm at time $t$ will yield a stream of expected monopoly profits, $v_{t+1}^{m}$, beginning in period $t+1$. With only one firm engaged in $R \& D$, equation (2.2) reduces to

$$
\kappa e^{-\kappa \ell_{t}} v_{t+1}^{m}=w_{t}
$$

Solving this for $\ell_{t}$ gives the amount of labor hired given the wage rate, $w_{t}$, the value of future profits, $v_{t+1}^{m}$, and the ease of $\mathrm{R} \& \mathrm{D}, \kappa$.

$$
\ell_{t}=-\frac{1}{\kappa} \ln \left(\frac{w}{\kappa v_{t+1}^{m}}\right)
$$

If more than one firm engages in $\mathrm{R} \& \mathrm{D}$ (i.e. $J>1$ ) and ties result in Bertrand competition in the product market, then a firm receives the stream of monopoly profits if and only if it is the only firm to innovate. In this case (2.2) becomes $\kappa e^{-\kappa \ell_{j}}\left[\left(e^{-\kappa \Sigma_{i \neq j} \ell_{i}}\right) v_{t+1}^{m}+\left(1-e^{-\kappa \Sigma_{i \neq j} \ell_{i}}\right) 0\right]=w$, where $\left(1-e^{-\kappa \Sigma_{i \neq j} \ell_{i}}\right)$ is the probability that at least one other firm innovates. This reduces to

$$
\kappa e^{-\kappa L} v_{t+1}^{m}=w
$$


where $L=\Sigma_{j} \ell_{j}$ is the aggregate employment by all R\&D firms.

Surprisingly, equations (5.1) and (5.2) yield the same aggregate employment, since $\ell=L$ when there is only one R\&D firm. Thus, a monopolistic innovator will hire the same amount of $R \& D$ as a group of innovators that expect to engage in Bertrand competition if there is an R\&D tie. Intuitively, all firms value an additional unit of labor only to the extent that it will succeed where all other units fail. For the monopolistic innovator this is because it owns any success from all other units anyway. For a member of a group this is because any success from units it does not own results in zero profits due to competition.

\section{Simple General Equilibrium}

A general equilibrium is a list of stochastic sequences $\left\langle\widetilde{\ell}_{1}, \widetilde{N}_{1}, \widetilde{C}_{1}, \widetilde{\mathbf{Q}}_{1}, \widetilde{r}_{1}, \widetilde{P}_{1}, \widetilde{w}_{1}, \widetilde{\pi}_{1}, \widetilde{\mathbf{q}}_{1}\right\rangle$ such that, for all $t$ and all realizations of the stochastic

process, (1) $\widetilde{\ell}_{1}$ is Nash equilibrium for innovators, (2) $\widetilde{C}_{1}$ and $\widetilde{\mathbf{Q}}_{1}$ solve the consumers' problem, (3) the full employment conditions $N_{t}+L_{t}=1$ are satisfied, (4) the credit market clearing conditions, that $Q_{i t}$ equal profits in state $t+1$ and state $i$ for all $t$ and $i$, are satisfied, and (5) the output price sequence $\widetilde{P}_{1}$ and the profit sequence $\widetilde{\pi}_{1}$ are derived as described above.

As is generally (though not universally ${ }^{5}$ ) the case in the Schumpeterian growth literature, we restrict attention to stationary equilibria. Consumption rises over time as new technologies are discovered. Let output in the first period be numeraire; that is $P_{1}=1$. The nature of equilibrium depends on the assumptions imposed on the market structure. We again consider three structures: (1) a single innovator, (2) multiple innovators that dissipate profits through Bertrand competition if more than one is successful, and (3) multiple innovators that successfully collude if more than one is successful. Of course, the first structure is a special case of either of the latter two. We refer to these, respectively, as the monopoly case, the Bertrand case, and the collusive case.

\footnotetext{
${ }^{5}$ See Deissenberg and Nyssen (1998).
} 


\subsection{Single Innovator}

When $J=1$, the successful innovator always becomes a monopolist. Posit a stationary equilibrium with a constant interest rate, $r$, and a constant output price, $P=1$. Then, since the monopoly price always prevails,

$$
P_{t+1}=\frac{w_{t+1} \theta}{A_{t+1}}=1
$$

for all $t$. Given an innovation in period $\mathrm{t}$, consumption will be constant at $C_{t+1}$ until further innovation occurs and (3.1) reduces to

$$
v_{t+1}^{s}=\left(\frac{1}{1+r-e^{-\kappa L}}\right)\left(\frac{\theta-1}{\theta}\right) C_{t+1}
$$

for all $t$. Now, by the definition of the output production function and the labor market clearing condition,

$$
C_{t+1}=A_{t+1}(1-L)
$$

for all $t$. Substituting the previous three relationships into the innovator's first order condition (2.2) and manipulating transforms it into

$$
e^{-\kappa L}[1+\kappa(\theta-1) \theta(1-L)]=1+r
$$

Equation (4.1) in this case is

$$
C_{t}^{-\sigma}=\beta(1+r) E\left\{C_{t+1}^{-\sigma}\right\}
$$

In a stationary equilibrium this implies

$$
1=\beta(1+r)\left[\rho \theta^{-\sigma}+(1-\rho)\right]
$$

This and the definition of $\rho$ imply

$$
(1+r)=\frac{1}{\beta\left[1+\left(1-e^{-\kappa L}\right)\left(\theta^{-\sigma}-1\right)\right]}
$$

Now (6.1) and (6.2) provide two equations in $r$ and $L$. The former exhibits $r$ decreasing in $L$ while the latter exhibits $r$ increasing in $L$. The unique solution will exist with $L>0$ if $\kappa \theta(\theta-1) \geq(1-\beta) / \beta$. The left side of this condition reflects the expected profitability of research while the right side reflects the degree to which future profits are discounted. Thus, if the likelihood of success (reflected in $\kappa$ ) and the gain from 
innovation (reflected in $\theta$ ) are sufficiently high and the future is not discounted too heavily (as reflected in $\beta$ ) then equilibrium will exhibit positive investment in research. Otherwise, $L=0$ in equilibrium and only (6.2) is satisfied, yielding no growth and an interest rate equal to the rate of time preference. The equilibrium values of the other variables can be derived in a straightforward manner.

\subsection{Multiple Innovators: Bertrand}

When $J>1$ R\&D ties will sometimes occur. When they do the structure of the equilibrium depends on producer behavior. This subsection assumes that multiple producers dissipate profits through Bertrand competition. Production in this case is greater than that with only one producer. This leads to cycles of monopoly and competition in the production sector which do not exist in the other market arrangements we consider.

Posit a stationary equilibrium exhibiting interest rates, $r^{M}$ and $r^{C}$ where the superscript $M$ denotes a variable's value when there is a monopoly producer and a $C$ denotes its value when production is competitive. As before, posit a constant output price, $P=1$. When a period- $t$ innovator, say innovator $j$, is the only successful firm, it enjoys monopoly profits until it is supplanted. In this case the output price satisfies

$$
P_{t+1}=w_{t+1}^{M} \theta / A_{t+1}=1 .
$$

If it is not the only successful firm, it and its Bertrand competitors will dissipate their profits by charging an output price (normalized to one) of ${ }^{6}$

$$
P_{t+1}=w_{t+1}^{C} / A_{t+1}=1
$$

Now, since consumption will be constant at $C_{t+1}$ for as long as the successful period- $t$ innovator is not supplanted, and since positive profits only accrue if innovator $j$ is the only successful firm, (3.2) reduces to

$$
v_{j, t+1}^{b k}=\frac{1}{1+r^{k}}\left(\frac{1+r^{M}}{1+r^{M}-e^{-\kappa L^{M}}}\right)\left(\frac{\theta-1}{\theta}\right) C_{t+1}^{M} ; k=M, C
$$

for all $t$. By the definition of the output production function and the labor market clearing condition,

\footnotetext{
${ }^{6}$ Since output is numeraire, the increased competition is reflected in a higher nominal wage, rather than a lower nominal output price.
} 


$$
C_{t+1}^{k}=A_{t+1}\left(1-L^{k}\right) ; k=M, C
$$

for all $t$. Substituting the previous four relationships into the innovator's first order condition (2.2) and manipulating yields

$$
\begin{aligned}
& e^{-\kappa L^{M}}\left[1+\kappa(\theta-1) \theta\left(1-L^{M}\right)\right]=1+r^{M} \\
& \kappa e^{-\kappa L^{C}}(\theta-1)\left(1-L^{M}\right) \frac{\left(1+r^{M}\right)}{\left(r^{M}+\rho^{M}\right)}=1+r^{C}
\end{aligned}
$$

There are two conditions because $\mathrm{R} \& \mathrm{D}$ employment and interest rates are different when current producers compete than it is when there is only one producer. Note that (6.3) \& (6.4) only determine aggregate R\&D employment, the distribution of investment across innovators is indeterminate. Of course, such indeterminacies are common in general equilibrium models with constant returns to scale, but this is not such a model. Intuitively, an innovator's marginal experiment is of value if and only if it succeeds when all other experiments fail. This is true whether the innovator or its competitors are conducting the other experiments; indeed as illustrated in section 5 , it is true whether or not the innovator has competitors. We assume below that all R\&D firms are equally sized: $\ell_{m j}=L_{m} / J, \ell_{c j}=L_{c} / J$ for all $i$

The consumer's Euler equations (4.2) can be written as

$$
\left(1+r^{M}\right)=\beta^{-1}\left[\rho_{0}^{M}+\rho_{1}^{M} \theta^{-\sigma}+\left(1-\rho_{0}^{M}-\rho_{1}^{M}\right)\left(\theta \frac{1-L_{C}}{1-L_{M}}\right)^{-\sigma}\right]^{-1}
$$

where $\rho_{0}^{M} \equiv e^{-\kappa L^{M}}, \rho_{1}^{M} \equiv J e^{-\kappa L^{M}(J-1) / J}\left(1-e^{-\kappa L^{M} / J}\right)$

$$
\left(1+r^{C}\right)=\beta^{-1}\left[\rho_{0}^{C}+\rho_{1}^{C}\left(\theta \frac{1-L_{M}}{1-L_{C}}\right)^{-\sigma}+\left(1-\rho_{0}^{C}-\rho_{1}^{C}\right) \theta^{-\sigma}\right]^{-1}
$$

where $\rho_{0}^{C} \equiv e^{-\kappa L^{C}}, \rho_{1}^{C} \equiv J e^{-\kappa L^{C}(J-1) / J}\left(1-e^{-\kappa L^{C} / J}\right)$

Equations (6.3) - (6.6) provide four equations in $L^{M}, L^{C}, r^{M} \& r^{C}$. Appendix 1 shows that this set of equations implies $L^{M}>L^{C}$ and $r^{M}>r^{C}$. Intuitively, when production is competitive, the real wage is higher than when there is a monopoly. This leads to a substitution of labor away from $R \& D$ and into production.

\subsection{Multiple Innovators: Collusion}

Now suppose that when multiple innovators are successful they collude and split the monopoly profits equally. Posit a stationary equilibrium with an interest rate of $r$, a 
constant output price, $P=1$, and symmetric investment, $\ell_{j}=L / J \forall j$. Then, since the monopoly price always prevails,

$$
P_{t+1}=\frac{w_{t+1} \theta}{A_{t+1}}=1
$$

for all $t$. Since consumption will be constant at $C_{t+1}$ for as long as the successful period- $t$ innovators are not supplanted, (3.3) reduces to

$$
v_{t+1}^{c}=\left(\frac{1}{1+r-e^{-\kappa L}}\right)\left(\frac{\theta-1}{\theta}\right) C_{t+1} \sum_{U \in 2^{J-1}}\left(\frac{\alpha(U)}{\# U+1}\right)
$$

Finally,

$$
C_{t+1}=A_{t+1}(1-L)
$$

Substituting these equations into the innovator's first order condition (2.2) and manipulating yields

$$
1+r=e^{-\kappa L}+\kappa e^{-\kappa L / J}(\theta-1) \theta(1-L) \sum_{i=0}^{J-1} \frac{1}{i+1} \frac{J !}{i !(J-i) !}\left(e^{-\kappa L / J}\right)^{(J-1-i)}\left(1-e^{-\kappa L / J}\right)^{i}
$$

Of course, when $J=1$, this corresponds to the monopoly case. The summed term is the expected inverse of the number of an innovator's successful competitors, and is declining in $L$. Thus the $r$ satisfying (6.7) is declining in $L$.

The consumer's Euler equation is the same as the monopoly case (6.2) and is reproduced here as (6.8).

$$
(1+r)=\frac{1}{\beta\left[1+\left(1-e^{-\kappa L}\right)\left(\theta^{-\sigma}-1\right)\right]}
$$

Equilibrium values of $L$ and $r$ solve (6.7) and (6.8). ${ }^{7}$ If there is a solution to (6.7) and (6.8) - that is, if $\kappa \theta(\theta-1) \geq(1-\beta) / \beta$ - then there is a unique stationary equilibrium and it exhibits a positive level of investment; otherwise, the unique stationary equilibrium exhibits no investment in R\&D.

Appendix 2 shows that $L$ is larger when $J>1$ than when $J=1$. Since $J=1$ is the case of a single innovator we examined above, it follows that more aggregate R\&D labor is hired with multiple innovators if collusive production is expected.

\footnotetext{
${ }^{7}$ As before, $L$ in equilibrium iff $\kappa \theta(\theta-1) \geq(1-\beta) / \beta$.
} 
It is easy to verify, given the properties of the binomial distribution imply $\Sigma_{j=0}^{J-1} \frac{\alpha(j)}{j+1}$ is declining in $J$, that an increase in the number of innovators $J$ decreases $\ell=L / J$ : faced with more competitors, each innovator invests less. ${ }^{8}$

\subsection{A social planner's problem}

Let $\mathbf{A}=\left\{A_{1}, \theta A_{1}, \theta^{2} A_{1}, \ldots\right\}$ be the set of attainable technology levels. A stationary social plan is a function $\lambda: \mathbf{A} \rightarrow[0,1]$ that assigns a level of investment to each level of technology. Define the social planner's problem as choosing a stationary social plan to maximize the expected present value of utility,

$$
E \sum_{t=1}^{\infty} \beta^{t-1} \frac{1}{1-\sigma} C_{t}^{1-\sigma}
$$

subject to the constraint that $\lambda(A) \in[0,1]$ for all $A \in \mathbf{A}$. Let $W(A, \lambda)$ be the expected present value of implementing the plan $\lambda$ if the current technology is $A$. Note that the choice of utility function implies $W\left(\theta A, \lambda^{\prime}\right)=\theta^{1-\sigma} W(A, \lambda)$ if $\lambda^{\prime}(\theta A)=\lambda(A)$ for all $A \in \mathbf{A}$. Let $V(A)=\max _{\lambda} W(A, \lambda)$. Standard continuity and compactness arguments imply that $V(A)$ is well defined if $1>\beta \theta^{1-\sigma}$, that is, if the maximal growth rate isn't too large relative to the rate of time preference. It follows from the properties of $W$ and the optimality of $V$ that $V(\theta A)=\theta^{1-\sigma} V(A)$. Let $\lambda^{*}$ be an optimal stationary plan. Then, for any $A \in \mathbf{A}$, standard recursive arguments imply

$$
V(A)=\frac{1}{1-\sigma}\left[A\left(1-\lambda^{*}(A)\right)\right]^{1-\sigma}+\beta\left[\rho\left(\lambda^{*}(A)\right) \theta^{1-\sigma} V(A)+\left(1-\rho\left(\lambda^{*}(A)\right)\right) V(A)\right]
$$

where, recall, $\rho(\ell)=1-e^{-\kappa L}$. Solving for $V(A)$ yields

$$
V(A)=\left(\frac{1}{1-\sigma}\right) \frac{A^{1-\sigma}\left(1-\lambda^{*}(A)\right)^{1-\sigma}}{1-\beta\left[\left(1-e^{-\kappa \lambda^{*}(A)}\right) \theta^{1-\sigma}+e^{-\kappa \lambda^{*}(A)}\right]}
$$

Since $V(A)$ is maximal, $\lambda^{*}(A)$ must maximize it for all $A$. Differentiating $V(A)$ with respect to $\lambda^{*}$, setting the result equal to zero, and manipulating it yields

$$
\frac{\beta \kappa e^{-\kappa \lambda^{*}(A)}\left(1-\lambda^{*}(A)\right)\left(\theta^{1-\sigma}-1\right)}{(1-\sigma)\left\{1-\beta\left[\left(1-e^{-\kappa \lambda^{*}(A)}\right) \theta^{1-\sigma}+e^{-\kappa \lambda^{*}(A)}\right]\right\}}=1
$$

\footnotetext{
${ }^{8}$ Graphically, an increase in $J$ shifts (6.4) and (6.5) to the left in $\ell-(1+r)$ space, illustrating the result
} 
Note that the left side of (6.9) is strictly decreasing in $\lambda^{*}(A)$, implying a unique optimal level of investment. Note also that the optimal level of investment is independent of $A$, that is, the social planner would optimize by choosing the same value for $L$ in every period.

Unfortunately, (6.9) cannot be explicitly solved for the optimal $L$ for purposes of comparison with the outcomes discussed in previous sections. Numerical simulations are tractable however. The next section presents some examples.

\section{Some numerical simulations}

It is well known that Schumpeterian growth models can exhibit either insufficient, optimal, or excessive growth relative to the social optimum. (See, for example, Aghion and Howitt (1998).) The intuition is compelling. On the one hand, successful innovators raise the entire future trajectory of the economy, but enjoy profits only until supplanted; this suggests growth rates will be too slow. On the other hand, successful innovators destroy the profitability of current producers, a social loss that innovators do not internalize; this suggests that growth rates will be too high. Either effect may dominate.

An implication of our analysis is that the welfare properties of equilibrium also depend on market structure. If there are at least two innovators, and if growth is insufficient in the Bertrand case, it can be increased toward (and even beyond) the optimal rate by allowing collusion. If growth is too high in the Bertrand case, allowing collusion worsens the problem. To provide a feel for the possible magnitudes of these effects, some simulations are reported in Table 2. For various parameter values, the table compares the social optimum, the monopoly outcome, Bertrand outcomes for various numbers of innovators, and the collusive outcome with the same numbers. In each simulation, imposing Bertrand competition in the case of ties substantially reduces growth below the social optimum. Further it reduces it below the level found in the monopoly case. Growth is significantly increased by allowing collusion even if there are only two innovators. In the last section we illustrate a case where collusion can even lead to R\&D levels higher than the social optimum.

\section{Concluding Remarks}


This paper has argued that post-innovation market structure matters in discretetime Schumpeterian growth models. Having a single innovator yields a socially suboptimal level of $R \& D$ and a growth rate that is too low. When the probability of simultaneous discoveries is non-negligible, having more than one innovator lowers aggregate growth rates if profits are dissipated by Bertrand competition in the event of a tie. By contrast, having multiple innovators can increase growth if they are allowed to collude in the event of a tie. The increased growth rate, which comes at the cost of additional R\&D expenditures, may or may not be welfare improving, but simulations suggest that allowing collusion may get it wrong by less than prohibiting collusion does.

The results of R\&D ties are usually not identical patents. We have modeled innovation as a discovery that lowers the cost of producing goods. It is just as easy to interpret innovation as an increase in quality of goods produced while cost remains constant. When ties occur in quality improvements the result will most likely be goods that are imperfect substitutes. In this case, the monopoly rents would not be completely dissipated and our results from the collusion case would apply.

Even if ties result in identical goods, however, it is possible that the collusive case is still the most relevant if patents are granted to only one firm. For example, if simultaneous discoveries are awarded to the first firm in line at the patent office, or by some random process, then the expected reward from a tie will be non-zero and the collusive case applies. 


\section{Appendix 1}

Multiple Innovators: Bertrand

We have four equations, (6.3)-(6.6) in four unknowns, $L^{M}, L^{C}, r^{M} \& r^{C}$

We can rewrite (6.4) as:

$$
e^{-\kappa L^{M}}\left[1+\kappa A(\theta-1)\left(1-L^{M}\right)\right]=1+r^{M} ; A \equiv\left(\frac{e^{-\kappa L^{C}}}{e^{-\kappa L^{M}}} \frac{1+r^{M}}{1+r^{C}}\right)
$$

Recall (6.3):

$e^{-\kappa L^{M}}\left[1+\kappa(\theta-1) \theta\left(1-L^{M}\right)\right]=1+r^{M}$

Hence, it must be that $A=\theta$.

We can use (6.5) and (6.6) to get:

$$
\frac{1+r^{M}}{1+r^{C}}=\frac{\rho_{0}^{C}+\rho_{1}^{C}\left(\theta \frac{1-L^{M}}{1-L^{C}}\right)^{-\sigma}+\left(1-\rho_{0}^{C}-\rho_{1}^{C}\right) \theta^{-\sigma}}{\rho_{0}^{M}+\rho_{1}^{M} \theta^{-\sigma}+\left(1-\rho_{0}^{M}-\rho_{1}^{M}\right)\left(\theta \frac{1-L^{C}}{1-L^{M}}\right)^{-\sigma}}
$$

and rewrite $\mathrm{A}$ as:

$$
A \equiv\left(\frac{e^{-\kappa L^{C}}}{e^{-\kappa L^{M}}} \frac{\rho_{0}^{C}+\rho_{1}^{C}\left(\theta \frac{1-L^{M}}{1-L^{C}}\right)^{-\sigma}+\left(1-\rho_{0}^{C}-\rho_{1}^{C}\right) \theta^{-\sigma}}{\rho_{0}^{M}+\rho_{1}^{M} \theta^{-\sigma}+\left(1-\rho_{0}^{M}-\rho_{1}^{M}\right)\left(\theta \frac{1-L^{C}}{1-L^{M}}\right)^{-\sigma}}\right)=\theta
$$

Recall the definitions, $\rho_{0}^{k} \equiv e^{-\kappa L^{k}}, \rho_{1}^{k} \equiv J e^{-\kappa L^{k}(J-1) / J}\left(1-e^{-\kappa L^{k} / J}\right) ; k=M, C$

Suppose $L^{M}=L^{C}$.

It is easy to see that this implies $A=1<\theta$.

Next, suppose $L^{M}<L^{C}$.

This implies $\rho_{0}^{M}>\rho_{0}^{C}$ and $\frac{1-L^{M}}{1-L^{C}}>1$.

It is possible to rewrite $A$ above as:

$$
A=\left(\frac{\rho_{0}^{C}}{\rho_{0}^{M}} \frac{\rho_{0}^{C}+\left(1-\rho_{0}^{C}\right) \theta^{-\sigma}-D^{C}}{\rho_{0}^{M}+\left(1-\rho_{0}^{M}\right) \theta^{-\sigma}+D^{M}}\right)
$$

where $D^{C} \equiv-\rho_{1}^{C}\left\{\theta^{-\sigma}\left[\left(\frac{1-L^{M}}{1-L^{C}}\right)^{-\sigma}-1\right]\right\}>0$, and $D^{M} \equiv\left(1-\rho_{0}^{M}-\rho_{1}^{M}\right)\left\{\theta^{-\sigma}\left[\left(\frac{1-L^{M}}{1-L^{C}}\right)^{\sigma}-1\right]\right\}>0$

Since $\theta^{-\sigma}<1$, we have $A<1<\theta$. 
Hence it must be that $L^{M}>L^{C}$.

In this case, $\rho_{0}^{M}<\rho_{0}^{C}$ and $\frac{1-L^{M}}{1-L^{C}}<1$, so $D^{C}$ and $D^{M}$ above are both negative.

This, in turn, implies that $r^{M}>r^{C}$. 


\section{Appendix 1}

Multiple Innovators: Collusion

Equation (6.7) is:

$$
\begin{aligned}
& 1+r=e^{-\kappa L}+\kappa e^{-\kappa L / J}(\theta-1) \theta(1-L) \sum_{i=0}^{J-1} \frac{1}{i+1} \frac{J !}{i !(J-i) !}\left(e^{-\kappa L / J}\right)^{(J-1-i)}\left(1-e^{-\kappa L / J}\right)^{i} \\
& 1+r=e^{-\kappa L}+\kappa(\theta-1) \theta(1-L) \sum_{i=0}^{J-1} \frac{1}{i+1} \frac{J !}{i !(J-i) !}\left(e^{-\kappa L / J}\right)^{(J-i)}\left(1-e^{-\kappa L / J}\right)^{i}
\end{aligned}
$$

Separating out the first term in the sum gives:

$$
\begin{gathered}
1+r=e^{-\kappa L}+\kappa(\theta-1) \theta(1-L)\left[e^{-\kappa L}+\sum_{i=1}^{J-1} \frac{1}{i+1} \frac{J !}{i !(J-i) !}\left(e^{-\kappa L / J}\right)^{(J-i)}\left(1-e^{-\kappa L / J}\right)^{i}\right] \\
1+r=e^{-\kappa L}[1+\kappa(\theta-1) \theta(1-L)]+A \\
A \equiv \kappa(\theta-1) \theta(1-L) \sum_{i=1}^{J-1} \frac{1}{i+1} \frac{J !}{i !(J-i) !}\left(e^{-\kappa L / J}\right)^{(J-i)}\left(1-e^{-\kappa L / J}\right)^{i}>0
\end{gathered}
$$

With $J=1 A=0$ and this reduces to (6.1) from the single innovator case

For a given level of $L, 1+r$ is higher in the case where $J>1$ than when $J=1$.

Since $r$ is decreasing in $L$ for (6.7) and increasing in $L$ for (6.8), moving from $J=1$ to $J>1$ will result in an increase in equilibrium $L$. As illustrated below:

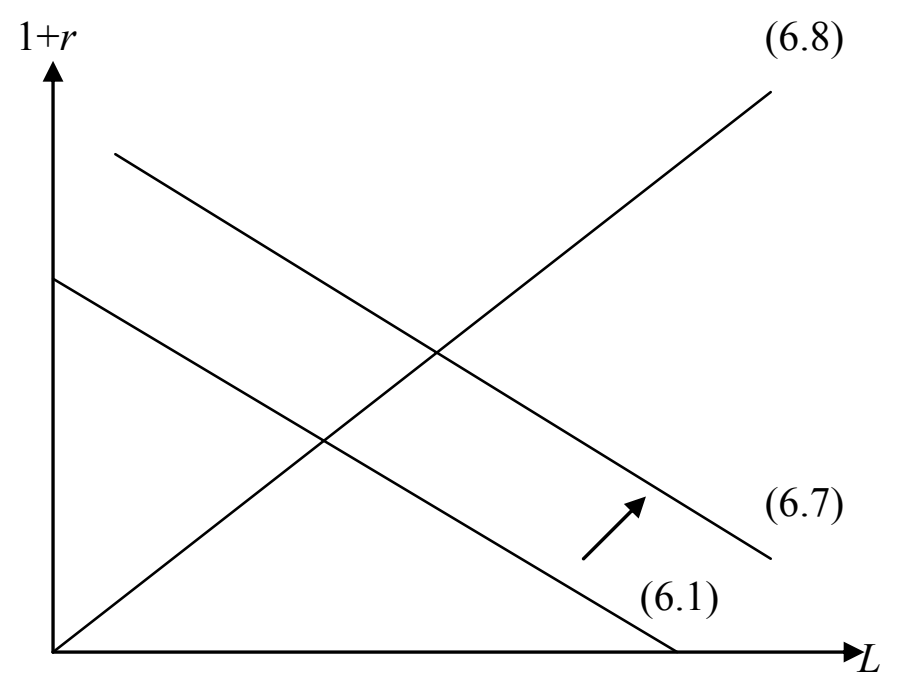




\section{Table 1}

Probabilities of Ties Inferred from Mean Technology Growth \& Markups

\begin{tabular}{|r|l|r|r|r|r|r|r|r|}
\hline $\begin{array}{r}\text { SIC } \\
\text { code }\end{array}$ & \multicolumn{1}{|c|}{ description } & $\boldsymbol{\theta}$ & $\boldsymbol{\theta}$ & $\begin{array}{c}\text { ties } \\
(\boldsymbol{J}=\mathbf{2})\end{array}$ & \multicolumn{1}{c|}{$\begin{array}{c}\text { ties } \\
(\boldsymbol{J}=\mathbf{5})\end{array}$} & \multicolumn{1}{c|}{$\begin{array}{c}\text { ties } \\
(\boldsymbol{J}=\mathbf{1 0})\end{array}$} & \multicolumn{1}{c|}{$\begin{array}{c}\text { ties } \\
(\boldsymbol{J}=\mathbf{1 0 0})\end{array}$} \\
\hline 20 & Food & $2.16 \%$ & 1.48 & $4.49 \%$ & $1.15 \%$ & $1.83 \%$ & $2.06 \%$ & $2.26 \%$ \\
\hline 22 & Textile mill products & $3.76 \%$ & 1.06 & $62.64 \%$ & $24.13 \%$ & $35.10 \%$ & $38.29 \%$ & $40.99 \%$ \\
\hline 23 & Apparel \& other textiles & $2.22 \%$ & 1.06 & $37.08 \%$ & $11.53 \%$ & $17.63 \%$ & $19.53 \%$ & $21.20 \%$ \\
\hline 24 & Lumber \& wood products & $2.13 \%$ & 1.10 & $21.29 \%$ & $5.98 \%$ & $9.34 \%$ & $10.43 \%$ & $11.39 \%$ \\
\hline 25 & Furniture \& fixtures & $1.47 \%$ & 1.10 & $14.68 \%$ & $3.97 \%$ & $6.25 \%$ & $6.99 \%$ & $7.65 \%$ \\
\hline 26 & Paper & $2.10 \%$ & 1.30 & $7.01 \%$ & $1.82 \%$ & $2.89 \%$ & $3.24 \%$ & $3.55 \%$ \\
\hline 28 & Chemicals & $3.55 \%$ & 3.12 & $1.67 \%$ & $0.42 \%$ & $0.67 \%$ & $0.76 \%$ & $0.83 \%$ \\
\hline 29 & Petroleum & $2.69 \%$ & 1.15 & $17.97 \%$ & $4.95 \%$ & $7.76 \%$ & $8.67 \%$ & $9.49 \%$ \\
\hline 30 & Rubber \& Plastics & $1.74 \%$ & 1.06 & $29.07 \%$ & $8.56 \%$ & $13.25 \%$ & $14.73 \%$ & $16.05 \%$ \\
\hline 32 & Stone, Clay \& Glass & $1.49 \%$ & 1.17 & $8.78 \%$ & $2.30 \%$ & $3.64 \%$ & $4.08 \%$ & $4.48 \%$ \\
\hline 33 & Primary Metals & $0.27 \%$ & 1.13 & $2.10 \%$ & $0.53 \%$ & $0.85 \%$ & $0.95 \%$ & $1.05 \%$ \\
\hline 34 & Fabricated Metals & $1.53 \%$ & 1.07 & $21.79 \%$ & $6.14 \%$ & $9.58 \%$ & $10.69 \%$ & $11.68 \%$ \\
\hline 35 & Machinery & $3.00 \%$ & 1.05 & $59.91 \%$ & $22.46 \%$ & $32.88 \%$ & $35.95 \%$ & $38.55 \%$ \\
\hline 36 & Electrical \& Electronic & $3.82 \%$ & 1.23 & $16.62 \%$ & $4.54 \%$ & $7.14 \%$ & $7.98 \%$ & $8.73 \%$ \\
\hline 37 & Transportation Equipment & $2.33 \%$ & 1.15 & $15.51 \%$ & $4.21 \%$ & $6.63 \%$ & $7.41 \%$ & $8.11 \%$ \\
\hline 38 & Instruments & $2.25 \%$ & 1.04 & $56.26 \%$ & $20.38 \%$ & $30.09 \%$ & $32.98 \%$ & $35.44 \%$ \\
\hline \hline & Average of All & $2.28 \%$ & 1.27 & $23.55 \%$ & $7.69 \%$ & $11.59 \%$ & $12.80 \%$ & $13.84 \%$ \\
\hline
\end{tabular}


Table 2

Results from Simulations

$\beta=0.95 \theta=1.05 \kappa=25 \sigma=0.75$

\begin{tabular}{|c|c|c|c|c|c|c|c|c|c|c|}
\hline \multirow[b]{2}{*}{ no. of R\&D firms } & \multirow{2}{*}{$\begin{array}{c}\text { Monopoly } \\
\mathrm{J}=1\end{array}$} & \multicolumn{4}{|c|}{ Bertrand } & \multicolumn{4}{|c|}{ Collusion } & \multirow[t]{2}{*}{ Optimum } \\
\hline & & $\mathrm{J}=2$ & $\mathrm{~J}=5$ & $\mathrm{~J}=10$ & $\mathrm{~J}=100$ & $\mathrm{~J}=2$ & $\mathrm{~J}=5$ & $\mathrm{~J}=10$ & $\mathrm{~J}=100$ & \\
\hline R\&D labor & $3.00 \%$ & $2.99 \%$ & $2.98 \%$ & $2.98 \%$ & $2.97 \%$ & $3.56 \%$ & $4.05 \%$ & $4.26 \%$ & $4.46 \%$ & $12.58 \%$ \\
\hline$\%$ optimal & $23.86 \%$ & $23.76 \%$ & $23.68 \%$ & $23.65 \%$ & $23.63 \%$ & $28.31 \%$ & $32.20 \%$ & $33.83 \%$ & $35.48 \%$ & $100.00 \%$ \\
\hline expected utility & 90.65 & 90.61 & 90.58 & 90.57 & 90.57 & 92.03 & 93.09 & 93.51 & 93.90 & 99.60 \\
\hline$\%$ welfare loss & $-8.98 \%$ & $-9.02 \%$ & $-9.05 \%$ & $-9.06 \%$ & $-9.07 \%$ & $-7.60 \%$ & $-6.53 \%$ & $-6.12 \%$ & $-5.72 \%$ & $0.00 \%$ \\
\hline avg. growth rate & $2.64 \%$ & $2.63 \%$ & $2.63 \%$ & $2.62 \%$ & $2.62 \%$ & $2.95 \%$ & $3.18 \%$ & $3.28 \%$ & $3.36 \%$ & $4.78 \%$ \\
\hline
\end{tabular}

$\beta=\mathbf{0 . 9 0} \theta=1.05 \kappa=25 \sigma=0.75$

\begin{tabular}{|c|c|c|c|c|c|c|c|c|c|c|}
\hline & Monopoly & \multicolumn{4}{|c|}{ Bertrand } & \multicolumn{4}{|c|}{ Collusion } & Optimum \\
\hline no. of R\&D firms & $\mathrm{J}=1$ & $\mathrm{~J}=2$ & $\mathrm{~J}=5$ & $\mathrm{~J}=10$ & $\mathrm{~J}=100$ & $\mathrm{~J}=2$ & $\mathrm{~J}=5$ & $\mathrm{~J}=10$ & $\mathrm{~J}=100$ & \\
\hline R\&D labor & $2.79 \%$ & $2.78 \%$ & $2.77 \%$ & $2.77 \%$ & $2.77 \%$ & $3.31 \%$ & $3.75 \%$ & $3.94 \%$ & $4.13 \%$ & $9.44 \%$ \\
\hline$\%$ optimal & $29.61 \%$ & $29.47 \%$ & $29.37 \%$ & $29.34 \%$ & $29.30 \%$ & $35.06 \%$ & $39.78 \%$ & $41.73 \%$ & $43.71 \%$ & $100.00 \%$ \\
\hline expected utility & 42.08 & 42.07 & 42.06 & 42.06 & 42.06 & 42.32 & 42.50 & 42.57 & 42.63 & 43.37 \\
\hline$\%$ welfare loss & $-2.98 \%$ & $-2.99 \%$ & $-3.00 \%$ & $-3.01 \%$ & $-3.01 \%$ & $-2.42 \%$ & $-2.00 \%$ & $-1.84 \%$ & $-1.69 \%$ & $0.00 \%$ \\
\hline avg growth rate & $2.51 \%$ & $2.51 \%$ & $2.50 \%$ & $2.50 \%$ & $2.50 \%$ & $2.81 \%$ & $3.04 \%$ & $3.13 \%$ & $3.22 \%$ & $4.53 \%$ \\
\hline
\end{tabular}

$\beta=0.95 \theta=\mathbf{1 . 2 0} \kappa=25 \sigma=0.75$

\begin{tabular}{|l|r|r|r|r|r|r|r|r|r|r|}
\hline & Monopoly & \multicolumn{4}{|c|}{ Bertrand } & \multicolumn{4}{|c|}{ Collusion } \\
\hline no. of R\&D firms & $\mathrm{J}=1$ & $\mathrm{~J}=2$ & $\mathrm{~J}=5$ & $\mathrm{~J}=10$ & $\mathrm{~J}=100$ & $\mathrm{~J}=2$ & $\mathrm{~J}=5$ & $\mathrm{~J}=10$ & $\mathrm{~J}=100$ & \\
\hline R\&D labor & $6.89 \%$ & $6.83 \%$ & $6.73 \%$ & $6.70 \%$ & $6.67 \%$ & $9.42 \%$ & $12.51 \%$ & $14.20 \%$ & $16.30 \%$ & $22.14 \%$ \\
\hline \% optimal & $31.12 \%$ & $30.86 \%$ & $30.41 \%$ & $30.27 \%$ & $30.14 \%$ & $42.53 \%$ & $56.49 \%$ & $64.14 \%$ & $73.62 \%$ & $100.00 \%$ \\
\hline expected utility & 298.16 & 294.21 & 289.70 & 288.31 & 287.12 & 404.22 & 514.53 & 558.87 & 597.69 & 641.18 \\
\hline \% welfare loss & $-53.50 \%$ & $-54.11 \%$ & $-54.82 \%$ & $-55.04 \%$ & $-55.22 \%$ & $-36.96 \%$ & $-19.75 \%$ & $-12.84 \%$ & $-6.78 \%$ & $0.00 \%$ \\
\hline avg growth rate & $16.43 \%$ & $16.34 \%$ & $16.24 \%$ & $16.21 \%$ & $16.19 \%$ & $18.10 \%$ & $19.12 \%$ & $19.43 \%$ & $19.66 \%$ & $19.92 \%$ \\
\hline
\end{tabular}


$\beta=0.95 \theta=1.05 \kappa=12.5 \sigma=0.75$

\begin{tabular}{|l|r|r|r|r|r|r|r|r|r|r|}
\hline & Monopoly & \multicolumn{4}{|c|}{ Bertrand } & \multicolumn{4}{|c|}{ Collusion } & Optimum \\
\hline no. of R\&D firms & $\mathrm{J}=1$ & $\mathrm{~J}=2$ & $\mathrm{~J}=5$ & $\mathrm{~J}=10$ & $\mathrm{~J}=100$ & $\mathrm{~J}=2$ & $\mathrm{~J}=5$ & $\mathrm{~J}=10$ & $\mathrm{~J}=100$ & \\
\hline R\&D labor & $3.42 \%$ & $3.39 \%$ & $3.38 \%$ & $3.37 \%$ & $3.37 \%$ & $3.80 \%$ & $4.09 \%$ & $4.20 \%$ & $4.30 \%$ & $18.67 \%$ \\
\hline \% optimal & $18.30 \%$ & $18.17 \%$ & $18.09 \%$ & $18.07 \%$ & $18.05 \%$ & $20.35 \%$ & $21.89 \%$ & $22.48 \%$ & $23.04 \%$ & $100.00 \%$ \\
\hline expected utility & 86.39 & 86.35 & 86.33 & 86.32 & 86.32 & 86.99 & 87.41 & 87.57 & 87.72 & 96.28 \\
\hline \% welfare loss & $-10.27 \%$ & $-10.31 \%$ & $-10.33 \%$ & $-10.34 \%$ & $-10.35 \%$ & $-9.66 \%$ & $-9.21 \%$ & $-9.04 \%$ & $-8.89 \%$ & $0.00 \%$ \\
\hline avg growth rate & $1.74 \%$ & $1.73 \%$ & $1.72 \%$ & $1.72 \%$ & $1.72 \%$ & $1.89 \%$ & $2.00 \%$ & $2.04 \%$ & $2.08 \%$ & $4.52 \%$ \\
\hline
\end{tabular}

$\beta=0.95 \theta=1.05 \kappa=25 \sigma=\mathbf{0 . 1 0}$

\begin{tabular}{|c|c|c|c|c|c|c|c|c|c|c|}
\hline & Monopoly & \multicolumn{4}{|c|}{ Bertrand } & \multicolumn{4}{|c|}{ Collusion } & Optimum \\
\hline no. of R\&D firms & $\mathrm{J}=1$ & $\mathrm{~J}=2$ & $\mathrm{~J}=5$ & $\mathrm{~J}=10$ & $\mathrm{~J}=100$ & $\mathrm{~J}=2$ & $\mathrm{~J}=5$ & $\mathrm{~J}=10$ & $\mathrm{~J}=100$ & \\
\hline R\&D labor & $3.07 \%$ & $3.03 \%$ & $3.02 \%$ & $3.01 \%$ & $3.01 \%$ & $3.65 \%$ & $4.17 \%$ & $4.38 \%$ & $4.60 \%$ & $14.48 \%$ \\
\hline$\%$ optimal & $21.19 \%$ & $20.95 \%$ & $20.83 \%$ & $20.80 \%$ & $20.77 \%$ & $25.23 \%$ & $28.77 \%$ & $30.27 \%$ & $31.79 \%$ & $100.00 \%$ \\
\hline expected utility & 39.81 & 39.57 & 39.45 & 39.41 & 39.39 & 43.96 & 47.77 & 49.42 & 51.13 & 113.62 \\
\hline$\%$ welfare loss & $-64.96 \%$ & $-65.17 \%$ & $-65.28 \%$ & $-65.31 \%$ & $-65.33 \%$ & $-61.31 \%$ & $-57.95 \%$ & $-56.50 \%$ & $-55.00 \%$ & $0.00 \%$ \\
\hline avg growth rate & $2.68 \%$ & $2.66 \%$ & $2.65 \%$ & $2.64 \%$ & $2.64 \%$ & $2.99 \%$ & $3.24 \%$ & $3.33 \%$ & $3.42 \%$ & $4.87 \%$ \\
\hline
\end{tabular}

\section{$\beta=0.95 \theta=\mathbf{2 . 0 0} \kappa=25 \sigma=\mathbf{0 . 9 5}$}

\begin{tabular}{|c|c|c|c|c|c|c|c|c|c|c|}
\hline \multirow[b]{2}{*}{ no. of R\&D firms } & \multirow{2}{*}{$\begin{array}{r}\text { Monopoly } \\
\mathrm{J}=1 \\
\end{array}$} & \multicolumn{4}{|c|}{ Bertrand } & \multicolumn{4}{|c|}{ Collusion } & \multirow[t]{2}{*}{ Optimum } \\
\hline & & $\mathrm{J}=2$ & $\mathrm{~J}=5$ & $\mathrm{~J}=10$ & $\mathrm{~J}=100$ & $\mathrm{~J}=2$ & $\mathrm{~J}=5$ & $\mathrm{~J}=10$ & $\mathrm{~J}=100$ & \\
\hline R\&D labor & $12.52 \%$ & $12.32 \%$ & $11.56 \%$ & $11.37 \%$ & $11.21 \%$ & $19.16 \%$ & $29.86 \%$ & $37.31 \%$ & $48.07 \%$ & $26.14 \%$ \\
\hline$\%$ optimal & $47.91 \%$ & $47.15 \%$ & $44.22 \%$ & $43.48 \%$ & $42.89 \%$ & $73.31 \%$ & $114.23 \%$ & $142.73 \%$ & $183.89 \%$ & $100.00 \%$ \\
\hline expected utility & 1129.84 & 1108.50 & 1094.80 & 1091.39 & 1088.69 & 1184.72 & 1189.92 & 1184.11 & 1173.16 & 1191.44 \\
\hline$\%$ welfare loss & $-5.17 \%$ & $-6.96 \%$ & $-8.11 \%$ & $-8.40 \%$ & $-8.62 \%$ & $-0.56 \%$ & $-0.13 \%$ & $-0.61 \%$ & $-1.53 \%$ & $0.00 \%$ \\
\hline avg growth rate & $95.63 \%$ & $94.28 \%$ & $93.36 \%$ & $93.13 \%$ & $92.95 \%$ & $99.17 \%$ & $99.94 \%$ & $99.99 \%$ & $100.00 \%$ & $99.85 \%$ \\
\hline
\end{tabular}




\section{References}

[1] Aghion, P. and P. Howitt (1992), "A model of growth through creative destruction," Econometrica, 60, pp. 323-351.

[2] Aghion, P. and P. Howitt (1998), Endogenous Growth Theory, MIT Press.

[3] Deissenbert, C. and J. Nyssen (1998),"A simple model of Schumpeterian growth with complex dynamics," Journal of Economic Dynamics and Control, 22, pp. 247-266/

[4] Grossman, G. and E. Helpman (1991), "Quality Ladders in the Theory of Growth," Review of Economic Studies, 63, pp. 43-61.

[5] Hall, R. (1988), "The Relation between Price and Marginal Cost in U.S. Industry." Journal of Political Economy, 96, pp. 921-947.

[6] Phillips, K. (1993), "Quality Ladders, Growth and R\&D: An Assessment from U.S. Industry," Carnegie-Rochester Conference Series on Public Policy, 38, pp. 239-274

[7] Segerstrom, P., T.C.A. Anant, and E. Dinopoulos (1990), "A Schumpeterian Model of the Product Life Cycle,” American Economic Review, 80, pp. 1077-1092. 